\title{
Quantum criticality
}

\author{
Piers Coleman ${ }^{1}$ \& Andrew J. Schofield ${ }^{2}$
}

${ }^{1}$ Center for Materials Theory, Rutgers University, Piscataway, New Jersey 08854-8019, USA (e-mail: coleman@physics.rutgers.edu)

${ }^{2}$ School of Physics \& Astronomy, University of Birmingham, Edgbaston, Birmingham B15 2TT, UK (e-mail:ajs@bham.ac.uk)

As we mark the centenary of Albert Einstein's seminal contribution to both quantum mechanics and special relativity, we approach another anniversary - that of Einstein's foundation of the quantum theory of solids. But 100 years on, the same experimental measurement that puzzled Einstein and his contemporaries is forcing us to question our understanding of how quantum matter transforms at ultra-low temperatures.

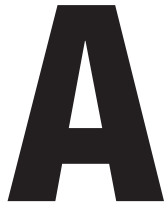

$\mathrm{t}$ the turn of the twentieth century a crisis less grandiose, yet just as profound as Michelson and Morley's failure to determine the Earth's motion through the ether, was troubling the founders of modern physics ${ }^{1}$. The puzzle stemmed, not from astrophysical observations, but from the innocent question about the amount of energy required to raise the temperature of diamond; that is, its specific heat. The trouble was that much less energy was needed than expected. Almost a hundred years before, Pierre-Louis Dulong and Alexis-Thérèse Petit had observed that no matter what a solid is made of, its specific heat per molecule is roughly the same. In 1876 this was put on an apparently solid theoretical foundation by Ludwig Boltzmann. By applying his statistical mechanics to the atoms in a solid he was able to compute the specific heat per atom in precise agreement with Dulong and Petit's early observations. But by the last years of the nineteenth century this triumph of statistical physics was looking increasingly hollow. Not only did some materials, such as diamond, show too small a specific heat but the advent of cryogenic techniques showed specific heats to be strongly temperature dependent at low temperatures, in contrast to Boltzmann's theory. Not for the last time would low-temperature physics point to the need for a new understanding.

Attempts to resolve the clash between theory and experiment were espoused by three great physicists of the nineteenth century: Boltzmann, Lord Kelvin and Lord Rayleigh. Boltzmann suggested that the way atoms behaved when confined within a solid might not be as straightforward as he had assumed. Lord Kelvin, on the other hand, looked to the mathematics of Boltzmann's derivation, convinced that the error lay there. It was Lord Rayleigh who was prepared to say that both the experiment and the theory were correct and that there was a true crisis which could only be resolved by fresh insight. The next step to solving the problem was made by Einstein.

\section{Einstein and the puzzle of specific heats}

Einstein took his inspiration from an unlikely source - the light from stars. In 1900 Max Planck had developed a formula that could relate the colour of stars to their temperature the first step in what would become quantum theory. Planck's theory had led Einstein to conclude that light is made up of discrete quanta or 'photons'. In 1906 Einstein applied Planck's formula to the vibrations inside matter which, he reasoned, must also be made up of quanta — tiny wave packets of sound that we now call 'phonons'. At high temperatures, Einstein's theory reverted to that of Boltzmann, but once the temperature dropped below that required to excite phonons, the heat content dropped dramatically. In his paper of November 1906 Einstein published one of his few fits to data - comparing his theory to the specific heat of diamond - to demonstrate his solution of a seventyyear-old puzzle. A new discipline of the quantum theory of solids was born.

Einstein's 1906 theory was necessarily incomplete but it contained the seeds of an impending revolution. A firstprinciples derivation of Einstein's simple theory had to wait until 1924 when it became the first problem to be solved by Werner Heisenberg using the new 'quantum mechanics'. By 1930 the approach pioneered by Einstein had essentially wrapped up the specific heat capacity problem.

\section{Quantum criticality and the return of the puzzle}

A century later, the very same measurement of the specific heat of solids points to a new clash with our theories of matter. The 'bad actors' of the twenty-first century are man-made crystals at the forefront of modern materials science. Physics has traditionally focused on stable phases of matter, such as those shown by superconductors, magnets or ferroelectrics, but with modern materials it is possible to study unstable quantum phases of matter. Puzzling new behaviour develops around the precarious point of instability between two stable phases of matter: the 'quantum critical point'. The unique properties that develop in quantum critical matter have become a major focus of research in the past ten years. Moreover, the discrepancies that are unfolding between established theory and experiment leave us in a state of affairs that is curiously similar to that which existed with diamond a hundred years ago.

As the materials of our lives become more sophisticated - from complex plastics to advanced metal ceramics one might think that understanding their properties would require knowing, in ever greater detail, the intricate complexities of electron and atom motion. Alas, such a task is impossible; even the most advanced computers are unable to cope with more than a few dozen electrons at once. Fortunately however, as Boltzmann foresaw when considering diamond, collections of particles can behave differently from isolated ones, and although their individual motions are complex, their collective properties acquire qualitatively new forms of simplicity. The appearance of magnetism, the development of zero-viscosity states in so-called superfluids, and the emergence of zero resistivity in a superconducting metal, are all examples of new, yet simple, behaviour that arise from the collective motion of electrons or atoms in complex matter. These are all examples of stable phases of matter. Collective behaviour also becomes important at the unstable interface between two stable phases of matter: the point of transformation is called a 'phase transition'.

Phase transitions are ubiquitous: from the crystalization of water into snowflakes, the alignment of electron spins inside a ferromagnet, the emergence of superconductivity in a cooled metal to the very formation of space-time in the early Universe; all involve phase transitions. Despite their 

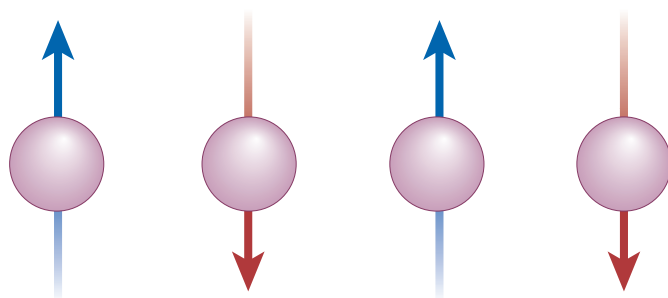

Figure 1 Staggered arrangement of spins in an antiferromagnet.

diversity, different phase transitions often share many fundamental characteristics. The specific heat when water turns to steam at a critical pressure has exactly the same power-law dependence on temperature as that of iron when it is demagnetized by having its temperature raised. Understanding this universal behaviour, known as 'critical phenomena', was a triumph of twentieth century physics'. One of the key discoveries was that the imminent arrival of order at such continuous phase transitions is signalled by the formation of short-lived droplets of nascent order that grow as the system is tuned to the critical point. At the critical point, the material is spanned by droplets of all sizes.

The melting of ice is, like most phase transitions, caused by the increase in random thermal motion of the molecules which occurs as the temperature is raised. The ordered arrangement of atoms that exists in the solid cannot be sustained beyond a certain temperature and the crystal melts. Yet research into condensed matter over the past decade has revealed a new kind of phase transition that is driven, not by thermal motion, but by the quantum fluctuations associated with Heisenberg's uncertainty principle. These quantum fluctuations are called 'zero-point motion'. According to the uncertainty principle, the more certain a particle's position, the more uncertain is its velocity. Thus, even when random, thermal motion ceases at the absolute zero temperature, atoms and molecules cannot be at rest because this would simultaneously fix their position and velocity. Instead they adopt a state of constant agitation. Like thermal motion, if zero-point motion becomes too wild, it can melt order, but in this case the melting takes place at absolute zero. Such a quantum phase transition $^{3}$ takes place in solid helium, which is so fragile that it requires a pressure to stabilize its crystal lattice even at absolute zero. When the pressure is released, zero-point motions melt the crystal.

The best studied examples of quantum phase transitions involve magnetism in metals. Electrons have a magnetic direction or spin, which when aligned in a regular fashion makes a material magnetic. Iron magnetizes when all the spins inside align in parallel, but in other materials the spins form a staggered, alternating, or antiferromagnetic, arrangement (Fig. 1). These more fragile types of order are more susceptible to melting by zero-point fluctuations. Almost three decades ago theoretical physicist John Hertz, now at Nordita, made the first study of how quantum mechanics would affect phase transitions ${ }^{4}$. Hertz was fascinated by the question of how critical phenomena might be altered by quantum mechanics. Applying quantum mechanics to phase transitions turns out to be very like Einstein's relativistic unification of space-time. In Hertz's theory, quantum mechanics appears by including a time dimension to the droplets of nascent order. Normally this produces no additional effect, but Hertz reasoned that if a phase transition took place at absolute zero, then the droplets of order that foreshadow the transition would become quantum-mechanical rather than classical. At a zero-temperature phase transition, he reasoned, these quantum droplets would grow to dominate the entire material, changing its properties in measurable ways - and most affected would be the electrons (Fig. 2). Such 'quantum critical matter' offers the real prospect of new classes of universal electronic behaviour developing independently of the detailed material behaviour, once the material is driven close to a quantum critical point ${ }^{5}$.
The electrons that carry current in a metal are similar to photons of light: they are quantum waves whose wavelength decreases as their momentum increases. Unlike photons however, electrons obey the 'Pauli exclusion principle': no two electrons can share the same wavelength (or momentum). To minimize their energy, each electron must occupy the momentum state with lowest energy that is not already filled. When all electrons have done this, there is then a sharp demarcation (called the Fermi surface) separating the highestenergy occupied and lowest-energy unoccupied momentum states. This ordering imposes severe constraints: like apples packed into a barrel, only those near the top can be easily rearranged. One consequence is seen in the specific heat: only electrons near the Fermi surface can absorb thermal energy and find an unoccupied momentum state to move to. This means that the specific heat of metals is tiny, but that it grows linearly with temperature. The coefficient of linearity, called the Sommerfeld coefficient, is a rough measure of the 'effective' mass of the highest-momentum electrons inside the metal. When the effective mass is compared to that of an isolated electron it is usually found to be somewhat larger. This is because of the forces between electrons, which mean that as an electron moves around it has to push neighbouring electrons aside.

a

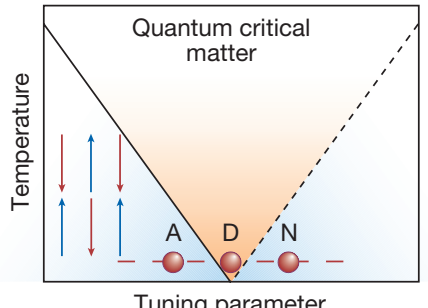

b

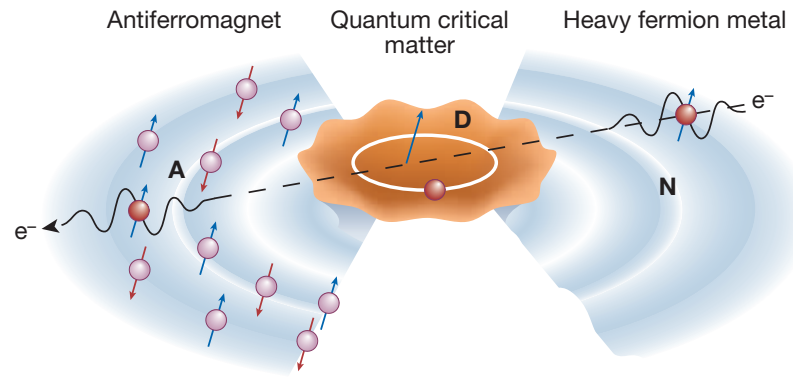

Figure 2 Schematic illustration of a quantum critical point showing the phase diagram (a) and the growth of droplets of quantum critical matter near the quantum critical point (b). a, Schematic phase diagram near a quantum critical point. Quantum critical points distort the fabric of the phase diagram creating a 'V-shaped' phase of quantum critical matter fanning out to finite temperatures from the quantum critical point. b, As matter is tuned to quantum criticality, ever-larger droplets of nascent order develop. On length-scales greater than these droplets, electrons propagate as waves. Inside the droplet, the intense fluctuations radically modify the motion of the electron, and may lead to it breaking up into its constituent spin and charge components. Physics inside the V-shaped region of the phase diagram (a) probes the interior of the quantum critical points (D), whereas the physics in the normal metal $(\mathrm{N}$ ) or antiferromagnet (A) reflects their exterior. If, as we suspect, quantum critical matter is universal, then no information about the microscopic nature of the material penetrates into the droplets. Making an analogy with a black hole, the passage from non-critical, to critical quantum matter involves crossing a 'material event horizon'. Experiments that tune a material from the normal metal past a quantum critical point force electrons through the 'horizon' in the phase diagram, into the interior of the quantum critical matter, from which they ultimately re-emerge through a second horizon on the other side into a new universe of magnetically ordered matter. 


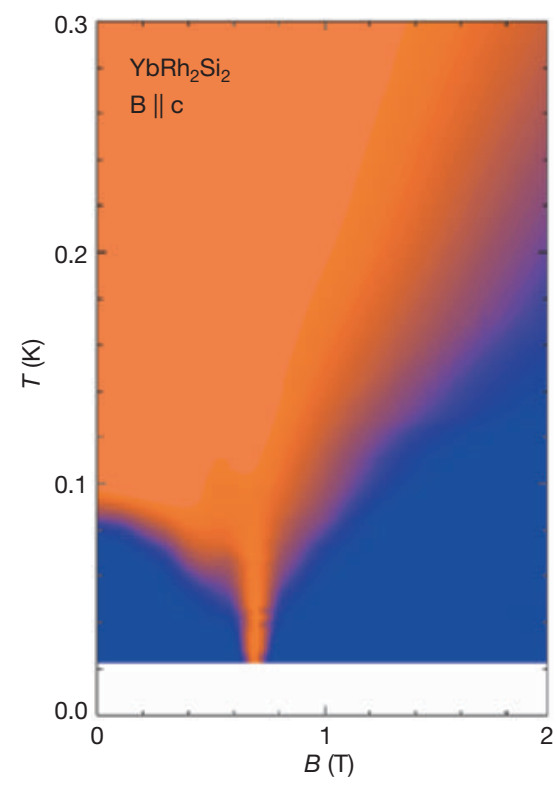

Figure 3 'Singularity in the phase diagram' illustrated by data taken from the material $\mathrm{YbRh}_{2} \mathrm{Si}_{2}$ where an applied magnetic field tunes the material to a quantum critical point ${ }^{25}$. Blue regions indicate normal metallic behaviour. Orange regions indicate anomalous metallic behaviour with linear resistivity. The singular quantum critical point at absolute zero produces a wide region of unusual metallic behaviour at finite temperatures.

But much more dramatic changes occur near a quantum phase transition. Ten years ago, Hilbert von Lohneyson's group in Karlsruhe, Germany, decided to measure the specific heat of a quantum critical metal $^{6}$. They chose $\mathrm{CeCu}_{6}$, which can be tuned to a magnetic - actually, an antiferromagnetic - quantum critical point by adding small amounts of gold. As they added gold to the metal, they found that the Sommerfeld ratio of the metal kept getting bigger - as if the approach to criticality led to a metal where the electrons were getting heavier and heavier. But at the quantum critical concentration, the Sommerfeld coefficient never settled down to a constant. It just kept on rising as they lowered the temperature, as if the mass of the electrons on the Fermi surface was becoming infinite and the energy of the electrons vanishing.

The Karlsruhe group found another disturbing property. In normal metals, the electrical resistance due to electrons scattering off one another grows as the square of the temperature, but in this system, at the quantum critical point, the resistivity is linear with temperature. The constancy of the Sommerfeld coefficient and the quadratic temperature dependence of the resistivity constitute two absolutely stalwart signatures of normal metals. Their breakdown suggests that a quantum critical metal is a fundamentally new type of electron fluid.

Since the original Karlsruhe measurements, many new discrepancies have come to light ${ }^{7}$. Quantum critical points have now been found by pressure tuning ${ }^{8}$ and by applying magnetic fields ${ }^{9}$. We even have an apparent case of a line of quantum criticality instead of a single point ${ }^{10}$. All support the notion that the characteristic energy scale of the metal is driven to zero at a quantum critical point. Indeed, temperature itself seems to be the only energy scale that remains in quantum critical matter. In neutron scattering ${ }^{11}$, for example, the rate at which electrons are scattered off the critical magnetic fluctuations seems to depend solely on the ratio of energy to temperature. Linear, or quasi-linear, resistivity is another sign of this phenomenon and, in the more dramatic cases, it can be followed over three decades in temperature.

One thing becoming increasingly clear is that although matter can never be cooled down to the quantum critical point at absolute zero, drastic effects are felt long before this point is reached (Figs 2 and 3). It is this influence that elevates quantum criticality from an intellectual abstraction at absolute zero to a real-world phenomenon that can profoundly change finite-temperature material properties. The quantum critical point represents a kind of 'black hole' in the material phase diagram and this proves a useful analogy. Just as the black hole of cosmology distorts surrounding space-time, quantum critical points distort the fabric of the phase diagram (Fig. 2), creating a ' $\mathrm{V}$ shaped' region of quantum critical matter fanning out to finite temperatures from the quantum critical point.

\section{Resolving the quantum critical enigma}

Some of the strange properties we see in quantum critical metals echo the predictions of Hertz and subsequent extensions of his theory ${ }^{12}$, but this conventional wisdom is flouted in at least two important respects. First, the effects are much stronger and wide ranging than Hertz expected. Second, the particular case of antiferromagnetism should be by far the least dramatic: the alternating pattern of up and down magnetism should average out for almost all electrons, slowing down only the tiny fraction that can interfere constructively with this arrangement. In marked contrast, the experiments tell us that the effective mass of every electron on the Fermi surface is being increased to infinity - in essence, bringing every electron to a halt. Like Einstein a century before us, we have a thirty-year-old theory that cannot account for present experiments.

The current theoretical alternatives also in some sense parallel those of Einstein's contemporaries, and there is great controversy. Some believe that the Hertz theory can indeed be saved if proper account is taken of the complexity of the material. The Karlsruhe team have advanced the idea that the underlying antiferromagnetic spin fluctuations at a quantum critical point collectively arrange themselves to become two-dimensional ${ }^{13}$. In this case, the motion of electrons between magnetic layers becomes highly turbulent, and it is possible to understand how they are driven to a halt in quantum critical matter. The only problem is that there is no obvious way for the spin fluctuations to become stubbornly two-dimensional in metals like $\mathrm{CeCu}_{6}$ where the electrons are not confined to layers.

Others seek to explain the discrepancy as a failure of Hertz's original approximations ${ }^{14}$. They point out that the distinction between the fluctuating magnetism and the electrons that appears in the Hertz theory is not so obvious when you remember that the magnetism itself also comes from the same electrons. Add to that the realities that materials contain disorder (atoms out of place, for example) and the assumptions of the mathematical derivations may not be quite so convincing.

Finally, there is the third possibility that, as Lord Raleigh advocated in the case of diamond, there is a true crisis and a new framework for quantum phase transitions is required. That would certainly be remarkable since the current theory is a beautiful synthesis of two very successful foundation stones of modern physics: the theory of temperature-driven phase transitions and quantum mechanics. Yet in the face of such puzzling experimental observations we are being driven towards such a possibility.

Several fascinating ideas have come to the forefront. One suggestion is that we are seeing a new kind of quantum criticality that turns classical criticality on its head. Classical criticality involves the global growth of static order in space: Hertz's theory takes this global model and also includes growth along the quantum time dimension. An alternative proposal is that quantum critical matter involves the growth of droplets of order in time, but not space ${ }^{15}$. The debate between proponents of 'global' and this 'local' quantum critical theory is highly contentious. Another possibility is that electrons break up inside the highly collective environment of quantum critical matter. It could be that electrons inside quantum critical matter break up into their constituent spin and charge components ${ }^{16}$ — like atoms splitting up into ions in solution. This is related to 'deconfined criticality' which has been used to describe quantum critical points in magnetic, 
two-dimensional insulators ${ }^{17}$. The only problem is that no one yet knows how to apply these ideas in detail to truly three-dimensional quantum critical metals.

The puzzle of quantum criticality is tantalizingly important for material science because, quite frequently, electrons appear to preempt the intense critical fluctuations of a quantum critical point by re-organizing themselves at the last minute into a new stable phase of matter. The divergence of the effective masses of the electrons and the collapse of electron kinetic energies all point to the formation of a highly degenerate state at the quantum critical point making them very susceptible to transformation into alternative stable electronic configurations.

For this reason, quantum criticality may be a highly effective catalyst for the formation of new stable types of material behaviour, providing an important new route for the design and discovery of new classes of material. One tendency is towards superconductivity ${ }^{18,19}$. High-temperature superconductors — which currently become superconducting at liquid nitrogen temperatures and inspire the hope for one day achieving room-temperature superconductivityexhibit a linear resistivity like metals, up to their melting temperature. Some believe that high-temperature superconductors and a whole host of other superconductors with quasi-linear resistances in the normal state, are driven by quantum criticality. Paradoxically, it is hard to prove this hypothesis because any attempt to remove the surrounding order will, at the same time, destroy the quantum criticality. We are faced by what has been described as a 'quantum conundrum' ${ }^{4}$.

Many other unidentified phases also appear to develop around quantum critical points. In $\mathrm{Sr}_{3} \mathrm{Ru}_{2} \mathrm{O}_{7}$ (ref. 20) and $\mathrm{URu}_{2} \mathrm{Si}_{2}$ (ref. 21), magnetic fields are used to tune the materials to a quantum critical point. These magnetic fields are too strong to allow the electrons to use superconductivity to avoid the critical singularity. Yet, rather than face the quantum critical point itself, the electrons find another way out. Abrupt changes in the resistivity and even in the material shape signal that the electrons have adopted a new type of ordered state. Is this a new form of magnetic order ${ }^{22}$ or a re-arrangement of the electrons' flow ${ }^{23}$ or perhaps both? All we know is that the electrons are still mobile but the precise rules of their new collective motion are still to be fully characterized and understood.

A hundred years ago, Einstein looked to the stars for inspiration to understand the properties of cold, stable, quantum matter. Today, the mysterious properties of critically unstable quantum matter not only suggest a new pathway to new material design, but they also raise our hopes for a new link between matter in the laboratory and matter in the cosmos. It has recently been proposed that ideas of quantum criticality may link up with quantum gravity at the surface of a black hole $^{24}$. Moreover, the break-up of electrons inside quantum critical matter and the emergence of new forces closely parallels challenges faced by the particle physicist, who seeks to understand how new forces develop between particles as they break up at high energies. Just as the inspiration of photons from stars led Einstein to solve the mystery of specific heat in stable quantum matter a hundred years ago, some of the ideas now being considered for the properties of particles in the early Universe, such as gauge theory and supersymmetry, may be poised to reappear, right under our nose in the laboratory, as the solution to the specific heat problem in quantum critical matter.

\section{doi:10.1038/nature03279}

1. Pais, A. Subtle is the Lord: the Science and the Life of Albert Einstein Ch. 20. 389-401 (Oxford Univ, Press, Oxford, 1982).

2. Domb, C. The Critical Point: a Historical Introduction to the Modern Theory of Critical Phenomena (Taylor \& Francis, London, 1996).

3. Sachdev, S. Quantum Phase Transitions (Cambridge Univ. Press, New York, 1999)

4. Hertz, J. Quantum critical phenomena. Phys. Rev. B 14, 1165-1184 (1976).

5. Laughlin, R. B., Lonzarich, G. G., Monthoux, P. \& Pines, D. The quantum criticality conundrum. Adv. Phys. 50, 361-365. (2001).

6. von Lohneysen, H. et al. Non-Fermi-liquid behavior in a heavy-fermion alloy at a magnetic instability. Phys. Rev. Lett. 72, 3262-3265 (1994).

7. Stewart, G. R. Non-Fermi-liquid behavior in d-and f-electron metals. Rev. Mod. Phys. 73, 797-855 (2001).

8. Julian, S. R. et al. The normal states of magnetic $d$ and $f$ transition metals. J. Phys. Condens. Matt. 8, 9675-9688 (1996).

9. Grigera, S. A. et al. Magnetic field tuned quantum criticality in the metallic ruthenate $\mathrm{Sr}_{3} \mathrm{Ru}_{2} \mathrm{O}_{7}$. Science 294, 329-332 (2001).

10. Doiron-Leyraud, N. et al. Fermi liquid breakdown in the paramagnetic phase of a pure metal. Nature 425, 595-599 (2003).

11. Schröder, A. et al., Onset of antiferromagnetism in heavy-fermion metals. Nature 407, 351-355 (2000).

12. Millis, A. J. Effect of a non-zero temperature on quantum critical points in itinerant fermion systems. Phys. Rev. B 48, 7183-7196 (1993).

13. Rosch, A. Interplay of disorder and spin fluctuations in the resistivity near a quantum critical point. Phys. Rev. Lett. 82, 4280-4283 (1999).

14. Belitz, D., Kirkpatrick, T. R. \& Rollühler, J. Breakdown of the perturbative renormalization group at certain quantum critical points. Phys. Rev. Lett. 93, 155701/1-4 (2004).

15. Si, Q., Rabello, S., Ingersent K. \& Smith, J. L. Locally critical quantum phase transitions in strongly correlated metals. Nature 413, 804-808 (2001).

16. Coleman, P., Pépin, C., Qimiao Si \& Ramazashvili, R. How do Fermi liquids get heavy and die? J. Phys. Condens. Matt. 13, R723-R738 (2001).

17. Senthil, T., Vishwanath, A., Balents, L., Sachdev, S. \& Fisher, M. P. A. Deconfined quantum critical points. Science 303, 1490-1494 (2004).

18. Mathur, N. D. et al. Magnetically mediated superconductivity in heavy fermion compounds. Nature 394, 39-43 (1998).

19. Petrovic, C. et al. A new heavy-fermion superconductor CeIrIn/sub 5/: a relative of the cuprates? Europhys. Lett. 53, 354-359 (2001).

20. Grigera, S. A. et al. Disorder-sensitive phase formation linked to metamagnetic quantum criticality. Science 306, 1154-1157 (2004).

21. Kim, K. H., Harrison, N., Jaime, M., Boebinger, G. S. \& Mydosh, J. A. Magnetic-field-induced quantum critical point and competing order parameters in $\mathrm{URu}_{2} \mathrm{Si}_{2}$. Phys. Rev. Lett. 91, 256401/1-4 (2003)

22. Amitsuka, H. et al. Hidden order and weak antiferromagnetism in $\mathrm{URu}_{2} \mathrm{Si}_{2}$. Physica B 312-3, 390-396 (2002).

23. Chandra, P. et al. Hidden orbital order in $\mathrm{URu}_{2} \mathrm{Si}_{2}$. Nature 417, 831-834 (2002).

24. Chapline, G. \& Laughlin, R. B. in Artificial Black Holes (eds Novello, M. et al.) 179-198 (World Scientific, Singapore, 2002).

25. Custers, J. et al. The break up of heavy electrons at a quantum critical point. Nature 424, 524-527 (2003).

Acknowledgements We gratefully acknowledge discussions with P. Chandra, Z. Fisk, A. P. Mackenzie and D. Pines. P.C. is supported by the National Science Foundation. A.J.S. is supported by the Royal Society, the Leverhulme Trust and the EPSRC.

Competing interests statement The authors declare that they have no competing financial interests. 